\title{
A Note on Surfaces in Space Forms with Pythagorean Fundamental Forms
}

\author{
Muhittin Evren Aydin 1,+(D) and Adela Mihai ${ }^{2, *,+}$ (D) \\ 1 Department of Mathematics, Firat University, 23000 Elazig, Turkey; meaydin@firat.edu.tr \\ 2 Department of Mathematics and Computer Science, Technical University of Civil Engineering, Bucharest, \\ 020396 Bucharest, Romania \\ * Correspondence: adela.mihai@utcb.ro \\ + The authors contributed equally to this work.
}

Received: 18 February 2020; Accepted: 13 March 2020; Published: 19 March 2020

check for updates

\begin{abstract}
In the present note we introduce a Pythagorean-like formula for surfaces immersed into 3-dimensional space forms $\mathbb{M}^{3}(c)$ of constant sectional curvature $c=-1,0,1$. More precisely, we consider a surface immersed into $\mathbb{M}^{3}(c)$ satisfying $\mathrm{I}^{2}+\mathrm{II}^{2}=\mathrm{III}^{2}$, where $\mathrm{I}$, II and III are the matrices corresponding to the first, second and third fundamental forms of the surface, respectively. We prove that such a surface is a totally umbilical round sphere with Gauss curvature $\varphi+c$, where $\varphi$ is the Golden ratio.
\end{abstract}

Keywords: Pythagorean formula; Golden ratio; Gauss curvature; space form

MSC: Primary: 11C20; Secondary: 11E25, 53C24, 53C42.

\section{Introduction and Statements of Results}

Let $\mathbb{N}^{*}$ denote set of all positive integers. For $a, b, c \in \mathbb{N}^{*}$, let $\{a, b, c\}$ be a triple with $a^{2}+b^{2}=c^{2}$, called a Pythagorean triple. The Pythagorean theorem states that the lengths of the sides of a right triangle turns to a Pythagorean triple. Moreover, if $\{a, b, c\}$ is a Pythagorean triple, so is $\{k a, k b, k c\}$, for any $k \in \mathbb{N}^{*}$. If gcd $(a, b, c)=1$, the triple $\{a, b, c\}$ is called a primitive Pythagorean triple. Of course, the most famous one among them is $\{3,4,5\}$. The Indian mathematician Brahmagupta (598-665 AD) gave a practical way generating all primitive Pythagorean triples: a triple $\left\{m^{2}-n^{2}, 2 m n, m^{2}+n^{2}\right\}$ is a primitive Pythagorean triple for every $m, n \in \mathbb{N}^{*}$ satisfying the following conditions

1. $m>n$,

2. $\operatorname{gcd}(m, n)=1$,

3. $m+n \equiv 1(\bmod 2)($ see $[1])$.

Recently, in [2], the authors extended this notion to the triple of integer-valued $n \times n$ matrices. Namely, a triple of such matrices $\{A, B, C\}$ is said to be Pythagorean if it satisfies

$$
\mathrm{A}^{2}+\mathrm{B}^{2}=\mathrm{C}^{2}
$$

As a trivial example, Equation (1) holds for any triple

$$
\left\{\mathrm{A}=\operatorname{diag}\left[a_{1}, \ldots, a_{n}\right], \mathrm{B}=\operatorname{diag}\left[b_{1}, \ldots, b_{n}\right], \mathrm{C}=\operatorname{diag}\left[c_{1}, \ldots, c_{n}\right]\right\}
$$

in which $\left\{a_{i}, b_{i}, c_{i}\right\}(i=1, \ldots, n)$ are Pythagorean triples. We refer to [2] for non-trivial examples and more details. We notice that this is not the first connection between Pythagorean triples and square matrices, see $[3,4]$. 
This interesting extension of Pythagorean triples motivates us to search a counterpart, in Differential Geometry, of this topic of Number Theory. For this purpose, a surface $M^{2}$ immersed into a 3-dimensional Riemannian space form $\mathbb{M}^{3}(c), c=-1,0,1$, satisfying

$$
\mathrm{I}^{2}+\mathrm{II}^{2}=\mathrm{III}^{2},
$$

where $\mathrm{I}^{2}, \mathrm{II}^{2}$ and $\mathrm{III}^{2}$ are the squares of the matrices corresponding to the first, second and third fundamental forms of $M^{2}$, respectively, is considered. We call Equation (2) the Pythagorean-like formula for a surface immersed into $\mathbb{M}^{3}(c)$.

As an example, let $\mathbb{M}^{3}(c)$ be the 3-dimensional Euclidean space $\mathbb{E}^{3}$, i.e., $c=0$. As usual we denote by $\mathbb{S}^{2}(r)$ a sphere of radius $r$ in $\mathbb{E}^{3}$ centered at the origin. As is known, the metric of $\mathbb{S}^{2}(r)$ is given by $\langle,\rangle_{I}=d u^{2}+\cos ^{2}\left(\frac{u}{r}\right) d v^{2}$; for $r \rightarrow \infty$ one naturally obtains the Euclidean metric $d u^{2}+d v^{2}$. The second and the third fundamental forms of $\mathbb{S}^{2}(r)$ are $h=-\frac{1}{r}\langle,\rangle_{I}$ and $\chi=\frac{1}{r^{2}}\langle,\rangle_{I}$. Therefore, $\mathbb{S}^{2}(r)$ satisfies the Pythagorean-like formula if and only if the following algebraic equation of degree 2 holds

$$
x^{2}+x-1=0,
$$

where $x=r^{2}$. Equation (3) has only one positive root, i.e., $x=\frac{\sqrt{5}-1}{2}$, which is the conjugate of $\varphi$, the Golden Ratio. This immediately implies that the Gauss curvature $K=1 / r^{2}$ of $\mathbb{S}^{2}(r)$ becomes the Golden Ratio.

Besides the Pythagorean Theorem, since the early ages, Golden ratio $\varphi$ $\left(\varphi=\frac{1+\sqrt{5}}{2}=1.61803398874989 \ldots\right)$ have had great interest not only for mathematicians but also for other scientists, philosophers, architects, and artists, for example see [5]. Indeed, we can see its importance due to Johannes Kepler (1571-1630), reference ([6]).

"Geometry has two great treasures; one is the Theorem of Pythagoras; the other, the division of a line into extreme and mean ratio. The first we may compare to a measure of gold; the second we may name a precious jewel".

The main result is the following.

Theorem 1. Let $M^{2}$ be a compact surface immersed into $\mathbb{M}^{3}(c), c=-1,0,1$, with nonzero extrinsic curvature everywhere. If $M^{2}$ satisfies a Pythagorean-like formula given by Equation (2), then it is a totally umbilical round sphere with Gauss curvature $\varphi+c$, where $\varphi$ is the Golden ratio.

Remark 1. For $c=1$, we take $\mathbb{M}^{3}(c)$ an open hemisphere $\mathbb{S}_{+}^{3}$.

We also denote by A the matrix corresponding to the shape operator A. The Pythagorean-like formula also can be interpreted in terms of shape operator A as

$$
\mathcal{I}^{2}+(\mathcal{I A})^{2}=\left(\mathcal{I A}^{2}\right)^{2},
$$

which is similar to the equation

$$
\mathcal{I}+\mathrm{A}=\mathrm{A}^{2},
$$

where $\mathcal{I}$ is identity on the tangent bundle of $M^{2}$. In [7], Equation (4) was completely solved for the so-called golden-shaped hypersurfaces in real space forms.

We notice that the starting point for the main idea of this study is the Pythagorean Theorem in spite of the fact that the Pythagorean-like formula given by Equation (2) is not directly related to the distance between points as in the usual case.

\section{Preliminaries}

In this section we provide some basics from $[8,9]$. 
Let $\mathbb{M}^{3}(c)$ denote a 3-dimensional Riemannian space form of constant sectional curvature $c=$ $-1,0,1$ and $\langle$,$\rangle a Riemannian metric on \mathbb{M}^{3}(c)$. Therefore, $\mathbb{M}^{3}(c)$ turns to the Euclidean space $\mathbb{E}^{3}$, the 3-sphere $\mathbb{S}^{3}$ and the hyperbolic space $\mathbb{H}^{3}$ when $c=0, c=1$ and $c=-1$, respectively. Here, $\mathbb{S}^{3}$ is the usual unit sphere of $\mathbb{E}^{4}$ given by

$$
\mathbb{S}^{3}=\left\{x=\left(x_{1}, x_{2}, x_{3}, x_{4}\right) \in \mathbb{E}^{4}:\langle x, x\rangle=1\right\},
$$

and $\mathbb{H}^{3}$ the hyperquadric of the Lorentz-Minkowski space $\mathbb{E}_{1}^{4}$ given by

$$
\mathbb{H}^{3}=\left\{x=\left(x_{1}, x_{2}, x_{3}, x_{4}\right) \in \mathbb{E}_{1}^{4}:\langle x, x\rangle_{L}=-1\right\},
$$

where $\langle,\rangle_{L}$ is the standard Lorentzian metric. We denote by $\mathbb{S}_{i,+}^{3}$ the open hemisphere consisting of all points $x$ on $\mathbb{S}^{3}$ with $x_{i}>0$.

Next, let $M^{2}$ be an orientable surface immersed into $\mathbb{M}^{3}(c)$ with metric $\langle,\rangle_{I}$ induced from Riemannian metric $\langle$,$\rangle on \mathbb{M}^{3}(c)$. Denote by $v$ the unit normal vector field over $M^{2}$ and $T_{p} M^{2}$ the tangent space of $M^{2}$ at the point $p$. For $x, y \in T_{p} M^{2}$, the second fundamental form is the symmetric bilinear form given by

$$
h_{p}(x, y)=\langle d v(x), y\rangle_{I}=\left\langle\mathrm{A}_{p}(x), y\right\rangle_{I},
$$

where A is the shape operator. $M^{2}$ is called totally geodesic when $h=0$ and totally umbilical when $h=\lambda\langle,\rangle_{I}$, where $\lambda$ is a nonzero constant. The eigenvalues of $\mathrm{A}$ at $p$, denoted by $\kappa_{1}$ and $\kappa_{2}$, are called the principal curvatures of $M^{2}$ at $p$. Denoting the trace of $\mathrm{A}$ by $\operatorname{tr}(\mathrm{A}), H(p)=\operatorname{tr}\left(\mathrm{A}_{p}\right) / 2=\left(\kappa_{1}+\kappa_{2}\right) / 2$ is called the mean curvature of $M^{2}$ at $p . M^{2}$ is said to be minimal if $H$ vanishes identically.

The Gauss equation for $M^{2}$ gives the Gauss curvature K by

$$
K=K_{\text {ext }}+c,
$$

where $K_{\text {ext }}$ is the extrinsic curvature of $M^{2}$, i.e., $K_{\text {ext }}=\operatorname{det} \mathrm{A}=\kappa_{1} \kappa_{2}$. In the Euclidean setting, obviously we have $K=K_{\text {ext }}$.

Noting that $\mathrm{A}$ is a self-adjoint linear operator at each point of $\mathrm{M}^{2}$, we introduce the third fundamental form of $M^{2}$ at $p$ by

$$
\chi_{p}(x, y)=\left\langle\mathrm{A}_{p}(x), \mathrm{A}_{p}(y)\right\rangle_{I} .
$$

Therefore, the Cayley-Hamilton Theorem for the matrix A has the form:

$$
\mathrm{III}-2 H \cdot \mathrm{II}+K_{\text {ext }} \cdot \mathrm{I}=0 .
$$

\section{Proof of Theorem 1}

Let $M^{2}$ be an immersed surface into $\mathbb{E}^{3}, \mathbb{H}^{3}$, or $\mathbb{S}_{+}^{3}$, respectively, satisfying the Pythagorean-like formula given by Equation (2). If $M^{2}$ is totally geodesic, i.e., II $=0$, then it follows III $=0$ and hence the Pythagorean-like formula leads to the contradiction I $=0$. Furthermore, if II is degenerate, or equivalently det II $=0$, then the Equations (2) and (5) imply

$$
\mathrm{I}^{2}=\left(4 H^{2}-1\right) \mathrm{II}^{2}
$$

which contradicts the fact that I is positive definite. Therefore, we necessarily assume det II $\neq 0$ everywhere. In the Euclidean setting, it is equivalent to assume $K \neq 0$ everywhere. If $M^{2}$ is minimal, from Equations (2) and (5) we derive

$$
\left(K_{e x t}^{2}-1\right) \mathrm{I}^{2}=\mathrm{II}^{2} .
$$


Taking the determinant, we obtain

$$
K_{e x t}^{4}-3 K_{e x t}^{2}+1=0,
$$

at each point of $M^{2}$. Then $K_{\text {ext }}$ is a nonzero constant, or equivalently, $K$ is constant. If the ambient space is $\mathbb{E}^{3}$ or $\mathbb{H}^{3}$ then $M^{2}$ must be totally geodesic (see [10] (Corollary 1)), which gives a contradiction. Otherwise, i.e., the ambient space is $\mathbb{S}_{+}^{3}$, there exist two cases (for details, see [11] (Corollary 3)):

Case a. $K=1$ and $M^{2}$ is totally geodesic. This case is not possible, already we discussed it above.

Case $\mathbf{b}$. $K=0$ and $M^{2}$ is an open piece of the Clifford torus. Thus, $K_{\text {ext }}=-1$, which does not fulfill Equation (7).

Consequently, an immersed surface into $\mathbb{E}^{3}, \mathbb{H}^{3}$, or $\mathbb{S}_{+}^{3}$ satisfying the Pythagorean-like formula can be neither totally geodesic, nor minimal, nor have degenerate second fundamental form.

Next we present the proof of the main result.

Proof of Theorem. Let $M^{2}$ be a compact surface immersed into $\mathbb{E}^{3}, \mathbb{H}^{3}$, or $\mathbb{S}_{+}^{3}$, respectively, with non-degenerate second fundamental form. Assume that $M^{2}$ satisfies the Pythagorean-like formula. By substituting (5) into (2), we get

$$
\left(1-K_{e x t}^{2}\right) \mathrm{I}^{2}+2 K_{e x t} H \mathrm{I} \cdot \mathrm{II}+2 K_{\text {ext }} H \mathrm{II} \cdot \mathrm{I}+\left(1-4 H^{2}\right) \mathrm{II}^{2}=0 .
$$

Notice that matrices do not commute by matrix multiplication ". ". Since I is positive definite and everywhere det II $\neq 0$, I and II have inverse matrices and thus Equation (8) can be rewritten as

$$
\mathrm{I}\left[\left(1-4 H^{2}\right) \mathrm{I}^{-1} \cdot \mathrm{II}+2 H K_{\text {ext }} \mathcal{I}_{2}\right] \mathrm{II}=\mathrm{II}\left[\left(K_{e x t}^{2}-1\right) \mathrm{II}^{-1} \cdot \mathrm{I}-2 H K_{\text {ext }} \mathcal{I}_{2}\right] \mathrm{I},
$$

where $\mathrm{I}^{-1}$ denotes the inverse matrix of $\mathrm{I}$ and $\mathcal{I}_{2}$ is the $2 \times 2$ unit matrix. Taking the determinant of the Equation (9), we obtain

$$
\operatorname{det}\left[\left(1-4 H^{2}\right) \mathrm{I}^{-1} \mathrm{II}+2 H K_{\text {ext }} \mathcal{I}_{2}\right]=\operatorname{det}\left[\left(K_{\text {ext }}^{2}-1\right) \mathrm{II}^{-1} \cdot \mathrm{I}-2 H K_{\text {ext }} \mathcal{I}_{2}\right] \text {. }
$$

Because $\mathrm{I}^{-1} \cdot \mathrm{II}=\mathrm{A}$ and $\mathrm{II}^{-1} \cdot \mathrm{I}=\mathrm{A}^{-1}$, Equation (10) reduces to

$$
\begin{aligned}
& \left(1-4 H^{2}\right)^{2} \operatorname{det} \mathrm{A}+2 H K_{\text {ext }}\left(1-4 H^{2}\right) \operatorname{tr}(\mathrm{A})= \\
& =\left(\frac{K_{\text {ext }}^{2}-1}{K_{e x t}}\right)^{2} \operatorname{det} \mathrm{A}-2 H K_{\text {ext }}\left(\frac{K_{\text {ext }}^{2}-1}{K_{\text {ext }}}\right) \operatorname{tr}(\mathrm{A}) .
\end{aligned}
$$

By substituting $K_{\text {ext }}=\operatorname{det} \mathrm{A}$ and $\operatorname{tr}(\mathrm{A})=2 \mathrm{H}$ into Equation (11), we obtain

$$
4 H^{2}\left(K_{e x t}^{2}-K_{e x t}-1\right)=\frac{\left(K_{e x t}^{2}-K_{e x t}-1\right)\left(K_{e x t}^{2}+K_{e x t}-1\right)}{K_{e x t}} .
$$

Now assume that $K_{e x t}^{2}-K_{e x t}-1 \neq 0$ in Equation (12). Thereby Equation (11) reduces to

$$
4 H^{2}=\frac{K_{e x t}^{2}+K_{e x t}-1}{K_{e x t}} .
$$

Because of compactness of $M^{2}$, there exist a point $p \in M^{2}$ at which $K_{\text {ext }}$ is strictly positive, i.e., $K_{\text {ext }}(p)>0$ (see [12] (Theorem 13.36)). Furthermore, because $4 H^{2}(p) \geq 4 K_{\text {ext }}(p)$, Equation (13) yields

$$
3 K_{\text {ext }}^{2}(p)-K_{\text {ext }}(p)+1 \leq 0,
$$


which is not possible because the left-hand side of formula (14) is strictly positive: contradiction. This implies from Equation (12) that

$$
K_{\text {ext }}^{2}-K_{\text {ext }}-1=0,
$$

for each point of $M^{2}$. Solving Equation (15) yields that $K_{\text {ext }}$ is a constant $\pm \varphi$, where $\varphi$ is the Golden Ratio. Since $K_{\text {ext }}$ is strictly positive at least at a point on $M^{2}$, one leads to $K_{\text {ext }}=\varphi$. Therefore, we obtain $K=\varphi+c$, for $c=-1,0,1$. This completes the proof by the fact that every compact surface with $K=$ constant is a totally umbilical round sphere (see [13] (Theorem 1 )).

\section{Conclusions}

Surfaces immersed into space forms satisfying the Pythagorean-like formula given by Equation (2) were investigated. Of course, the roles of I and II in Equation (2) are symmetric. Moreover, the study of those surfaces satisfying the following equations could be challenging problems:

$$
\mathrm{I}^{2}+\mathrm{III}^{2}=\mathrm{II}^{2} \text { and } \mathrm{II}^{2}+\mathrm{III}^{2}=\mathrm{I}^{2} .
$$

Furthermore, the above Pythagorean-like formula given for surfaces can be extended to hypersurfaces (or submanifolds of codimension $>1$ ) in space forms.

Author Contributions: Conceptualization, M.E.A.; Investigation, M.E.A. and A.M.; Methodology, M.E.A. and A.M.; Supervision, A.M. All authors have read and agreed to the published version of the manuscript.

Funding: This research received no external funding.

Conflicts of Interest: The authors declare no conflict of interest.

\section{References}

1. Takloo-Bighash, R. A Pythagorean Introduction to Number Theory: Right Triangles, Sums of Squares, and Arithmetic; Springer: Cham, Switzerland, 2018.

2. Arnold, M.; Eydelzon, A. On matrix Pythagorean triples. Am. Math. Monthly 2019, 126, 158-160. [CrossRef]

3. Crasmareanu, M. A new method to obtain Pythagorean triple preserving matrices. Missouri J. Math. Sci. 2002, 14, 149-158.

4. Palmer, L.; Ahuja, M.; Tikoo, M. Finding Pythagorean triple preserving matrices. Missouri J. Math. Sci. 1998, 10, 99-105. [CrossRef]

5. Dunlap, R.A. The Golden Ratio and Fibonacci Numbers; World Scientific Publ. Co.: Hackensack, NJ, USA, 1997.

6. Livio, M. The Golden Ratio: The Story of Phi, the World's Most Astonishing Number; Broadway Books: New York, NY, USA, 2003.

7. Crasmareanu, M.; Hretcanu, C.-E.; Munteanu, M.-I. Golden- and product-shaped hypersurfaces in real space forms. Int. J. Geom. Methods Mod. Phys. 2013, 10, 1320006. [CrossRef]

8. Chen, B.-Y. Geometry of Submanifolds; M. Dekker: New York, NY, USA, 1973.

9. O'Neill, B. Semi-Riemannian Geometry with Applications to Relativity; Academic Press: New York, NY, USA, 1983.

10. Chen, B.-Y. Minimal surfaces with constant Gauss curvature. Proc. Am. Math. Soc. 1972, 34, 504-508. [CrossRef]

11. Lawson, H.B., Jr. Local rigidity theorems for minimal hypersurfaces. Ann. Math. 1969, 89, $187-197$. [CrossRef]

12. Gray, A. Modern Differential Geometry of Curves and Surfaces with Mathematica; CRC Press LLC: Boca Raton, FL, USA, 1998.

13. Aledo, J.A.; Alías, L.J.; Romero, A. A new proof of Liebmann classical rigidity theorem for surfaces in space forms. Rocky Mt. J. Math. 2005, 35, 1811-1824. [CrossRef]

(C) 2020 by the authors. Licensee MDPI, Basel, Switzerland. This article is an open access article distributed under the terms and conditions of the Creative Commons Attribution (CC BY) license (http://creativecommons.org/licenses/by/4.0/). 